\title{
ESTUDIO Y DESARROLLO DE UN SECADERO DE ADOBES DE LADRILLOS MACIZOS
}

Msc. R. SPOTORNO, D.E.A. J. POCHETTINO, O. PASERO, Ing. J. OHSE, Ing. F. GARCÍA (*)

\section{RESUMEN:}

Se presenta el estudio y desarrollo de un secadero de adobes de una fábrica de ladrillos macizos de la zona de Makalle (Chaco). Se obtuvieron curvas representativas del proceso de secado para una capacidad de 5000 adobes en el interior del secadero. Se logró determinar para una temperatura aproximada de $50^{\circ} \mathrm{C}$ del aire que ingresa al secadero, la cantidad de agua extraída de los adobes es de $6265 \mathrm{~kg}$ y el tiempo final del proceso de secado es de aproximadamente 60 horas. Además se seleccionaron los diferentes componentes del secadero como ser: ventiladores, economizador y bomba de calor.

Palabras claves: adobe, estudio y desarrollo, secadero

\section{INTRODUCCIÓN}

La importancia que ha adquirido el ladrillo a través de su historia lo ha colocado como un material indispensable en la industria de la construcción a nivel mundial. Muchas de las construcciones de albañilería que se realizan hoy en día tienen como componente básico al ladrillo, que en nuestro medio es elaborado mayormente de arcilla. Los ladrillos son pequeñas piezas cerámicas en forma de paralelepípedo, formadas por tierras arcillosas, moldeadas, comprimidas y sometidas a una cocción. Pueden utilizarse en toda clase de construcciones por ser su forma regular y fácil manejo (Moreno, 1981). La fabricación de ladrillos macizos en el nordeste argentino se ve afectada durante el periodo de abril a setiembre, debido a que se extiende demasiado el proceso de secado, llegando a deteriorarse en el caso de lluvias y tormentas. La totalidad de las ladrillerias del Chaco, ya sean las de procesos de corte manual o mecanizado, necesitan demasiados días para lograr el secado óptimo del adobe, antes de ingresar al proceso de cocción del mismo. Este inconveniente

(*) GITEA-Grupo de Investigación en Tecnologías Energéticas Apropiadas. Universidad Tecnológica Nacional. Facultad Regional Resistencia. French 414.3500. Resistencia-Chaco. República Argentina Tel: +54 3624 432928/Fax: +54 3624 432683/e-mail: rubenspotorno@yahoo.com 
y la gran demanda de este producto de gran importancia para la construcción de viviendas y edificios conllevan a la necesidad de reducir el tiempo de secado. La ladrilleria de corte mecanizado SICAMAR S.A, ubicada en la localidad de Makalle, posee una gran producción de ladrillos, presentando el inconveniente mencionado oportunamente respecto al secado de los adobes. Por tal motivo los propietarios de la ladrilleria solicitaron al G.I.T.E.A (Grupo de Investigación en Tecnologías Energéticas Apropiadas), asistencia técnica con la finalidad de resolver el problema de secado.

Actualmente a nivel mundial existen trabajos de investigación referidos a procesos de secado de adobes, pero la gran mayoría sobre ladrillos cerámicos. En cuanto al proceso de secado de adobes para la producción de ladrillos macizos existe bibliografía referida a las curvas de secado de Bigot (uniovi, 2016), las que muestran las etapas de eliminación del agua dependiendo del tipo de arcilla utilizada. El G.I.T.E.A presentó en el III Congreso Argentino de Ingeniería, los resultados experimentales obtenidos del proceso de secado de adobes de arcilla de la zona de Makalle (Chaco) (Spotorno, et al., 2016).

En el presente trabajo se exponen los resultados obtenidos del estudio y desarrollo de un secadero de adobes, que utiliza como fuente principal de energía los gases residuales de combustión de los hornos de cocción de los adobes y una fuente de energía alternativa mediante la utilización de una bomba de calor.

\section{MATERIALES Y MÉTODOS}

El secado de un cuerpo arcilloso crudo es el mecanismo por el cual se elimina el agua que lo humedece, el cual es necesario para que la cocción del adobe se realice adecuadamente. El mecanismo de secado es muy similar para los distintos cuerpos arcillosos. No obstante, a una determinada velocidad de secado, los efectos que se generan sobre cada cuerpo, pueden ser muy diferentes entre cada uno de ellos, dependiendo de su naturaleza química y cristalográfica, de su granulometría y de su historia previa antes de llegar al secadero (uniovi, 2016). Cabe aclarar que el estudio y desarrollo del secadero se realizó para una capacidad de 5000 adobes que poseen las siguientes dimensiones: largo: $260 \mathrm{~mm}$, ancho: $160 \mathrm{~mm}$ y espesor: $56 \mathrm{~mm}$, denominado comercialmente ladrillo de 20 .

Para realizar el estudio energético necesario para el desarrollo del secadero, se debió calcular la cantidad de agua extraída del mismo durante el proceso de secado de los adobes, con lo cual se realizaron curvas representativas de secado que se muestran a continuación.

En la figura 1 se observa la gráfica de las curvas de humedades absolutas de entrada y salida del aire en el secadero en función del tiempo. Se puede apreciar que en las primeras horas de secado las curvas se encuentran significativamente separadas y a medida que transcurre el tiempo las mismas tienden a juntarse (finalización del proceso de secado de los adobes). 


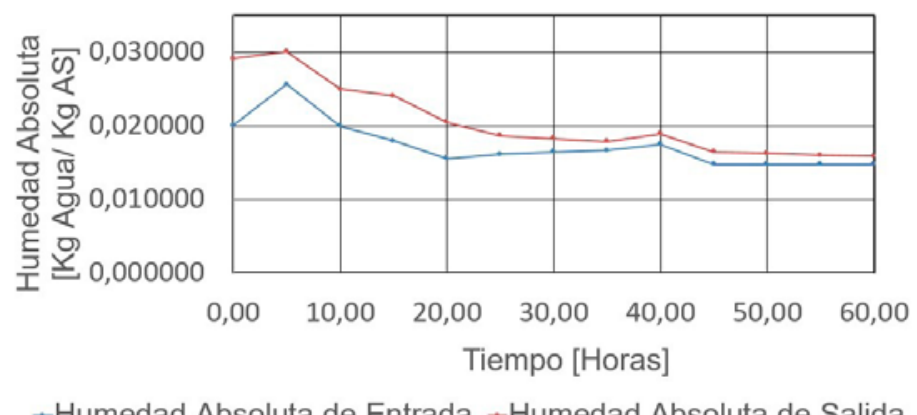

Figura 1: Humedades absolutas del aire

La gráfica de la figura 2 muestra el comportamiento de las temperaturas de entrada y salida del aire en el secadero. Se observa que para una temperatura de entrada del aire de $50^{\circ} \mathrm{C}$, al cabo de 60 horas la temperatura de salida del aire se aproxima a la de entrada, indicando que el proceso de secado de adobes finaliza.

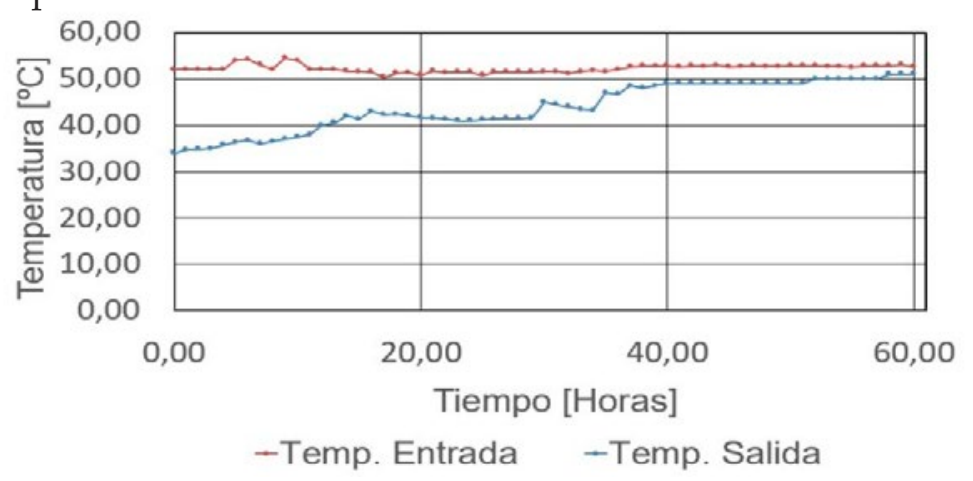

Figura 2: Temperaturas del aire

En la figura 3 se observa la pérdida de peso de los 5000 adobes en función del tiempo. Se puede apreciar que al cabo de 60 horas de secado, la pérdida de peso alcanza un valor aproximado de $6265 \mathrm{~kg}$, lo que representa una pérdida de agua de 1250 gramos cada adobe.

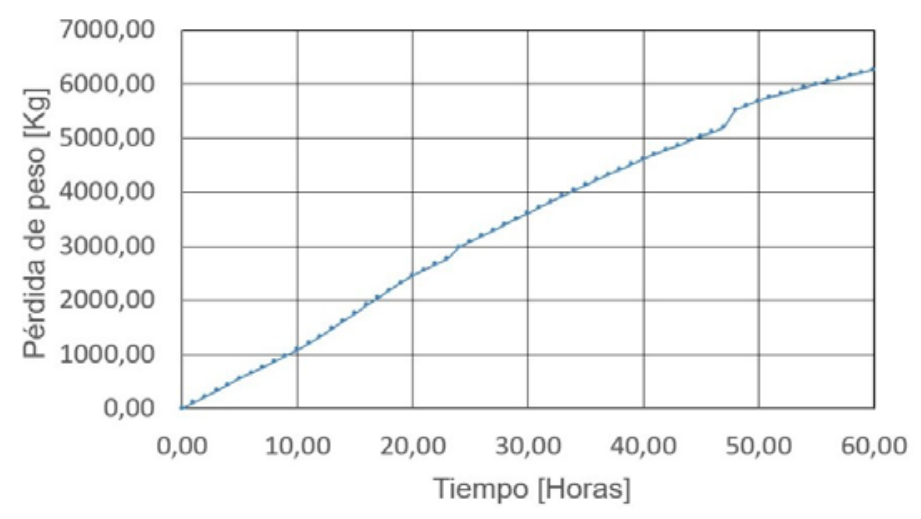

Figura 3: Pérdida de peso de los adobes 
La figura 4 muestra el proceso de secado de los adobes. En el eje de ordenada se representa la humedad en base seca, logrando apreciar que al finalizar el proceso de secado, el adobe concluye con un $12 \%$ de humedad al cabo de un periodo de 60 horas, condición óptima para ingresar al horno de cocción.

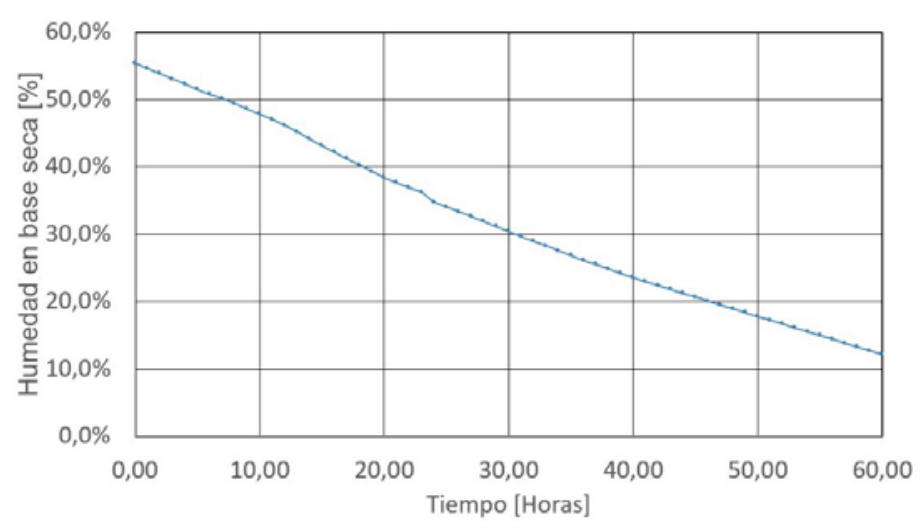

Figura 4: Proceso de secado de los adobes

\section{Determinación de parámetros termodinámicos}

En la figura 5 se observa un esquema del proceso de secado de los adobes. El proceso 1-2 es el calentamiento del aire realizado en el economizador, mediante la utilización de los gases residuales de combustión procedentes del horno de cocción o de la bomba de calor en el caso que no se realice la cocción de los adobes. El proceso 2-3 es la circulación del aire caliente a través del secadero, mientras que $(\mathrm{m}+\mathrm{w})$ es el adobe al inicio del proceso de secado y ms1 el adobe deshidratado

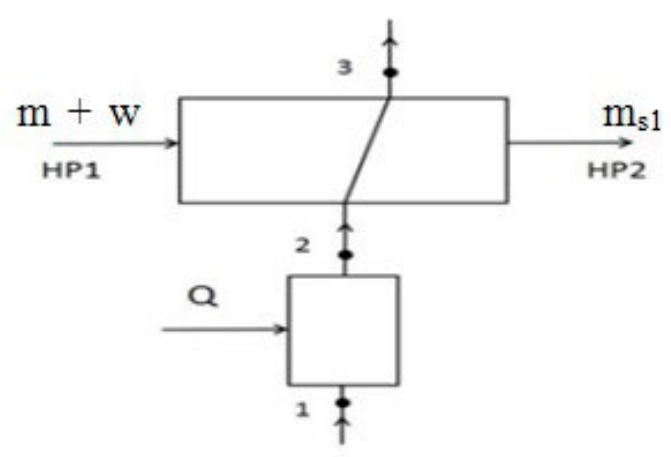

Figura 5: Esquema del proceso de secado

A continuación se presenta el cálculo de parámetros termodinámicos, que permiten seleccionar los componentes del secadero de adobes, que se mencionan a continuación: ventilador centrífugo y axial, economizador y bomba de calor. 


\section{Humedad del adobe en base seca}

La cantidad de agua con que finaliza el adobe después del proceso de secado, en referencia con la humedad en base seca, se determina mediante la fórmula (1).

$$
\begin{aligned}
& \mathrm{HP}_{2}= \\
& \frac{m_{s 1}-m_{s}}{m_{s}}
\end{aligned}
$$

Donde:

$\mathrm{HP}_{2}$ : humedad del adobe al finalizar el secado

$\mathrm{m}_{\mathrm{s}}$ : masa del ladrillo $(\mathrm{kg})$

$\mathrm{m}_{\mathrm{s} 1}:$ masa del adobe seco $(\mathrm{kg})$

\section{Cantidad de agua que debe extraerse}

$$
\begin{aligned}
& \frac{\Delta_{\mathrm{x}}=}{\mathrm{m}_{\mathrm{th}}-\mathrm{m}_{\mathrm{s} 1}} \\
& \mathrm{t}
\end{aligned}
$$

Donde:

$\Delta_{\mathrm{x}}:$ cantidad de agua a extraerse del secadero $(\mathrm{kg} / \mathrm{h})$

$\mathrm{m}_{\mathrm{th}}:$ masa total húmeda $(\mathrm{kg})$

$\mathrm{m}_{\mathrm{s} 1}:$ masa del adobe seco $(\mathrm{kg})$

t: tiempo (h)

Se considera masa del ladrillo, al adobe libre de humedad, que se retira del horno de cocción.

\section{Caudal másico de aire que debe ingresar al secadero}

$$
\begin{aligned}
& \dot{m}_{\mathrm{a}}= \\
& \frac{\left|\dot{\Delta}_{\mathrm{x}}\right|}{\left(\mathrm{x}_{3}-\mathrm{x}_{1}\right)}
\end{aligned}
$$

Donde:

$\dot{\mathrm{m}}_{\mathrm{a}}$ : caudal másico de aire seco $(\mathrm{kg} / \mathrm{h})$

$\left(\mathrm{X}_{3}-\mathrm{X}_{1}\right)$ : diferencia de humedad absoluta del aire entre la salida y la entrada al secadero $\left(\mathrm{kg}_{\text {agua }} / \mathrm{kg}_{\text {aire seco }}\right)$

$\left|\dot{\Delta_{\mathrm{x}}}\right|:$ cantidad de agua a extraer de los adobes $\left(\mathrm{kg}_{\text {agua }} / \mathrm{h}\right)$

El caudal volumétrico de aire que debe ingresar al secadero se determina mediante la fórmula (4).

$$
\begin{aligned}
& \dot{\mathbf{v}}= \\
& \dot{\mathbf{m}}_{\mathbf{a}} \cdot \mathbf{v}
\end{aligned}
$$


Donde:

$\dot{V}$ : caudal volumétrico de aire $(\mathrm{m} 3 / \mathrm{h})$

$\dot{m}_{\mathrm{a}}$ : caudal másico de aire seco $(\mathrm{kg} / \mathrm{h})$

$\mathrm{v}$ : volumen específico del aire $(\mathrm{m} 3 / \mathrm{kg})$

\section{Potencia calorífica para calentar el aire}

$$
\dot{\mathrm{Q}_{\mathrm{a}}}=\dot{\mathrm{m}}_{\mathrm{a}} \cdot \mathrm{Cp} \cdot\left(\mathrm{T}_{2}-\mathrm{T}_{1}\right)
$$

Donde $\dot{Q}_{a}$ es la potencia calorífica en $\mathrm{kW}, \mathrm{Cp}$ es el calor específico del aire en $(\mathrm{kJ} / \mathrm{kg} . \mathrm{K})$, $\mathrm{T}_{1}$ es la temperatura del aire exterior al ingreso del economizador en $(\mathrm{K}), \mathrm{y}_{2}$ es la temperatura del aire a la salida de la cámara de mezcla en (K). Ver figura 10.

\section{Desarrollo del secadero de adobes}

Considerando la disposición de los adobes en el interior del secadero, se determinó el volumen necesario que nos permitió el diseño del mismo. A continuación se observa un croquis y un esquema de los componentes del secadero.

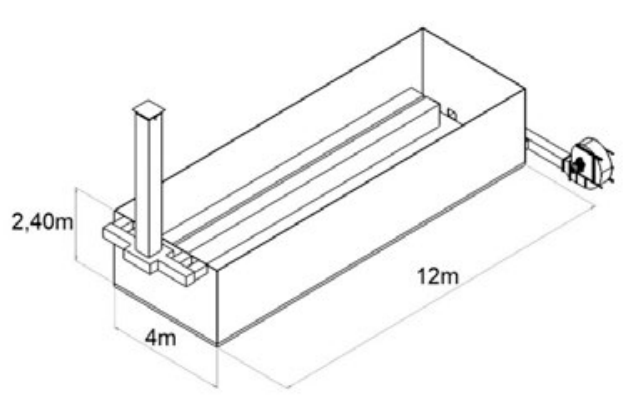

Figura 6: Dimensiones del secadero

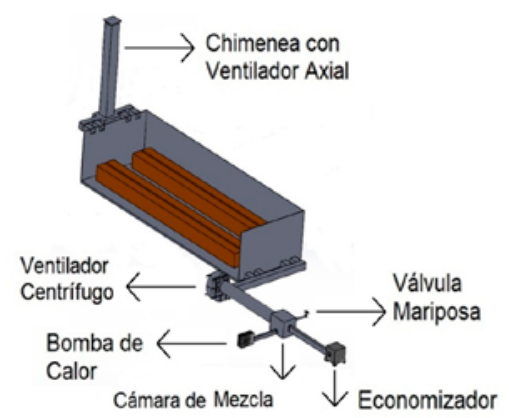

Figura 7: Componentes del secadero

Se puede observar en la figura 7 los diferentes componentes del secadero de adobes: economizador, ventilador centrífugo, bomba de calor, cámara de mezcla y chimenea con ventilador axial. Con la finalidad de desarrollar un secadero con bajas pérdidas de energía al exterior, se adoptó para el diseño de la cámara de secado, materiales con las siguientes características:

Para la construcción de paredes y techo se utilizaron paneles denominados comercialmente PANEL plac, que consiste en paneles termo-aislantes de 0,40 $\mathrm{m}$ de ancho por el largo a medida de cada faldón, conformados por cara exterior de chapa de acero (B) BWG galvanizada de Siderar primera calidad espesor 0,41 mm, un núcleo termo-aislante $(\mathrm{A})$ de poliestireno expandido EPS de $50 \mathrm{~mm}$ de espesor y una cara interior $(\mathrm{C})$ de MDF símil madera de $5 \mathrm{~mm}$ de espesor (terminación natural) apta para pintar o barnizar. Las características particulares de esta cubierta se pueden observar en (panelplac, 2017). 


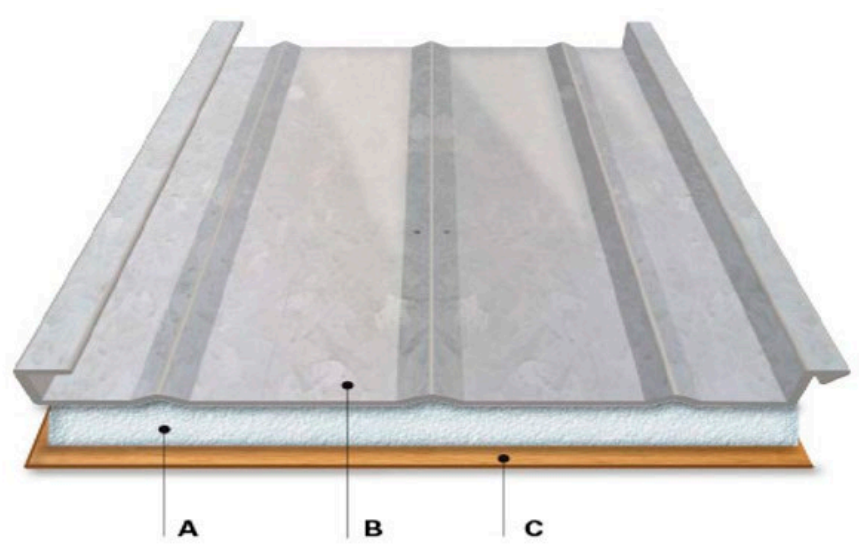

Figura 8: Módulo de PANELplac

Se optó por este sistema ya que permite generar módulos (secaderos) acorde a las dimensiones que se necesitan. En la figura 8 se observa la gráfica del módulo de PANELplac utilizado para el desarrollo del secadero de adobes.

\section{Simulación de la circulación del aire caliente en el secadero}

En el trabajo se realizaron simulaciones de los perfiles de velocidad, temperatura y presión, para observar el comportamiento de la circulación del aire caliente en el secadero. La simulación se realizó mediante el complemento Flow Simulation del programa SolidWorks 2016 y se establecieron las siguientes condiciones:

Se realizó la configuración de las dimensiones del volumen de control. Como condiciones de borde se establece que las paredes, techo, piso y ductos son adiabáticos. Para subdividir el volumen de control se consideró un mallado intermedio. La figura 9 muestra la simulación del perfil de velocidad de circulación del aire caliente.

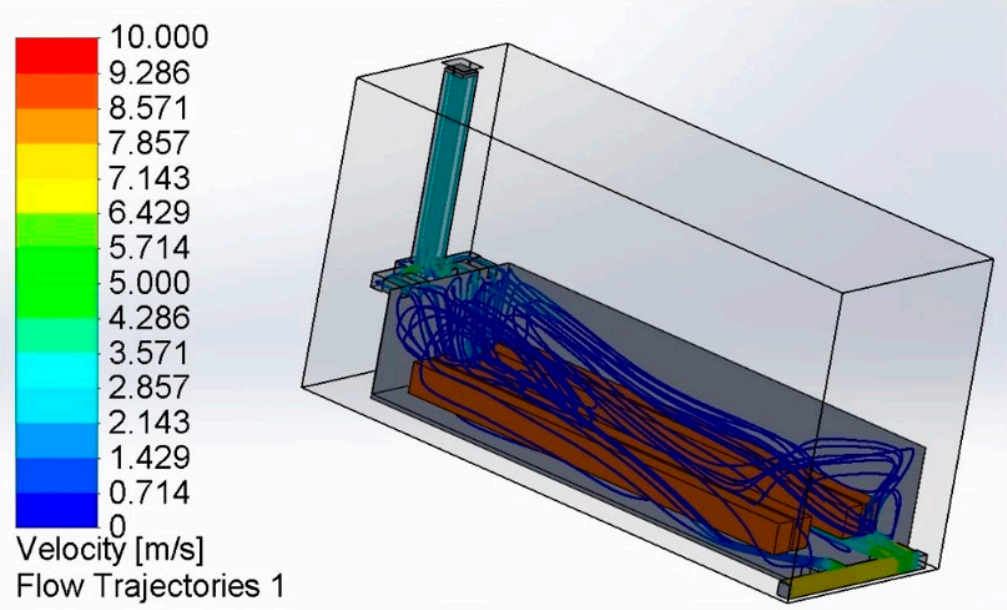

Figura 9: Simulación del perfil velocidad 


\section{Selección de los ventiladores}

$\mathrm{El}$ aire caliente que sale del economizador necesariamente debe ser impulsado a través de los adobes para producir el secado de los mismos. Un ventilador centrífugo impulsa el aire caliente circulando a lo largo del secadero, de manera que en el otro extremo del mismo se produce la salida al exterior mediante la aspiración producida por un ventilador axial colocado en la chimenea.

Considerando el caudal volumétrico de aire (formula 4), que debe circular por el secadero de adobes, se seleccionó un ventilador centrífugo marca GATTI (gattisa, 2017), con las siguientes características: Modelo RA390 (112M, 5.5 CV).

$\mathrm{El}$ aire caliente que pasa a través de los adobes durante el proceso de secado, se extrae del secadero mediante una chimenea y un ventilador axial marca GATTI (gattisa, 2017), con las siguientes características: Modelo KRT450/2-25 2 aspas $2 \mathrm{CV}$.

\section{Selección del economizador}

El proceso de secado de los adobes en el interior del secadero se realiza utilizando aire caliente. Si utilizaríamos los gases de combustión del horno de cocción para realizar el proceso de secado, se pondría en riesgo la salud de los operarios que trabajan en el interior del secadero. Por tal motivo el aire exterior se calienta mediante un economizador, por donde circulan los gases de combustión del horno de cocción y de esta

manera el aire caliente se introduce en el secadero de adobes.

La selección del economizador se fundamenta teniendo en cuenta la potencia calorífica determinada en la fórmula (5). En la figura 10 se observa el economizador seleccionado (solerpalau, 2017), y a continuación las características del mismo: CRMTC/4 225/090 0,55KW LG 270 230/400V 50HZ VE.

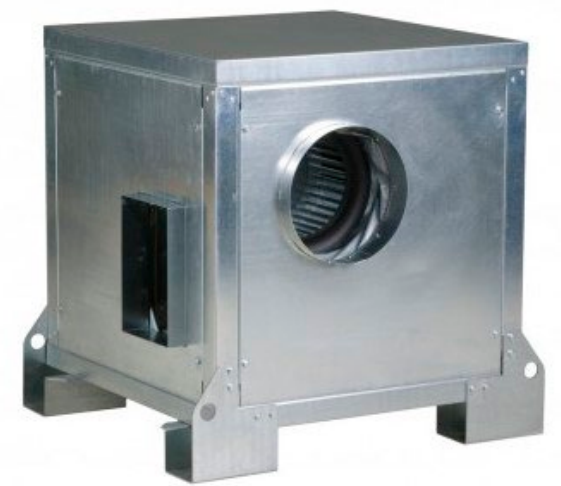

Figura 10: Economizador

\section{Selección de la bomba de calor}

La utilización de los gases de combustión para el calentamiento del aire que se emplea para el proceso de secado de los adobes, se ve interrumpida cuando no se realiza en 
los hornos la cocción de los adobes. Con el fin de evitar este inconveniente que produciría que el proceso de secado se paralice, se implementó la utilización de una fuente de energía adicional mediante una bomba de calor, de forma que sustituya la energía aportada por los gases de combustión.

La potencia calorífica necesaria para extraer el agua durante el proceso de secado de los adobes, permitió seleccionar la bomba de calor (climatecnica, 2017), con las siguientes características: Modelo 658EZ-140-SA SURREY Bomba de 12,5 TR.

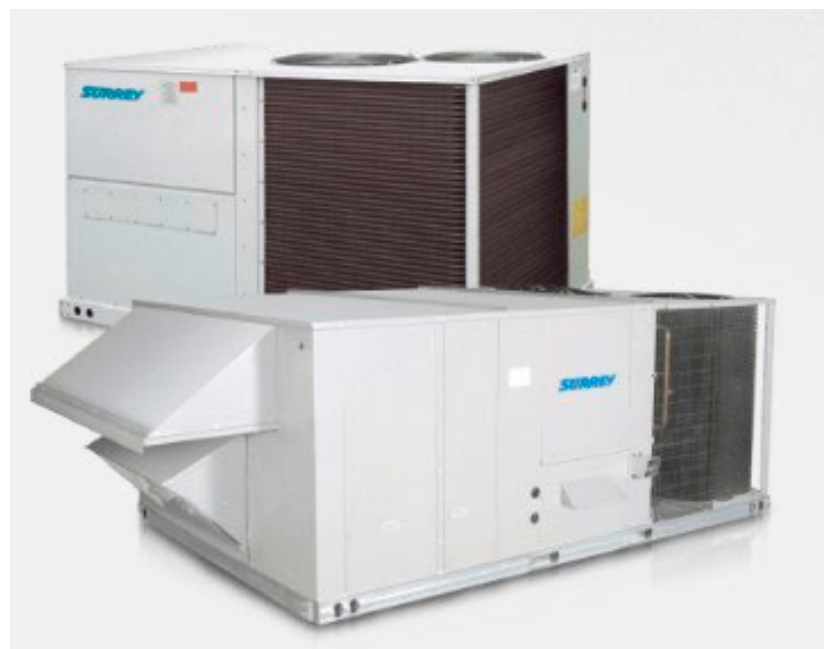

Figura 11: Bomba de calor

La figura 11 muestra la bomba de calor seleccionada.

Esquema funcional del sistema de calentamiento del aire

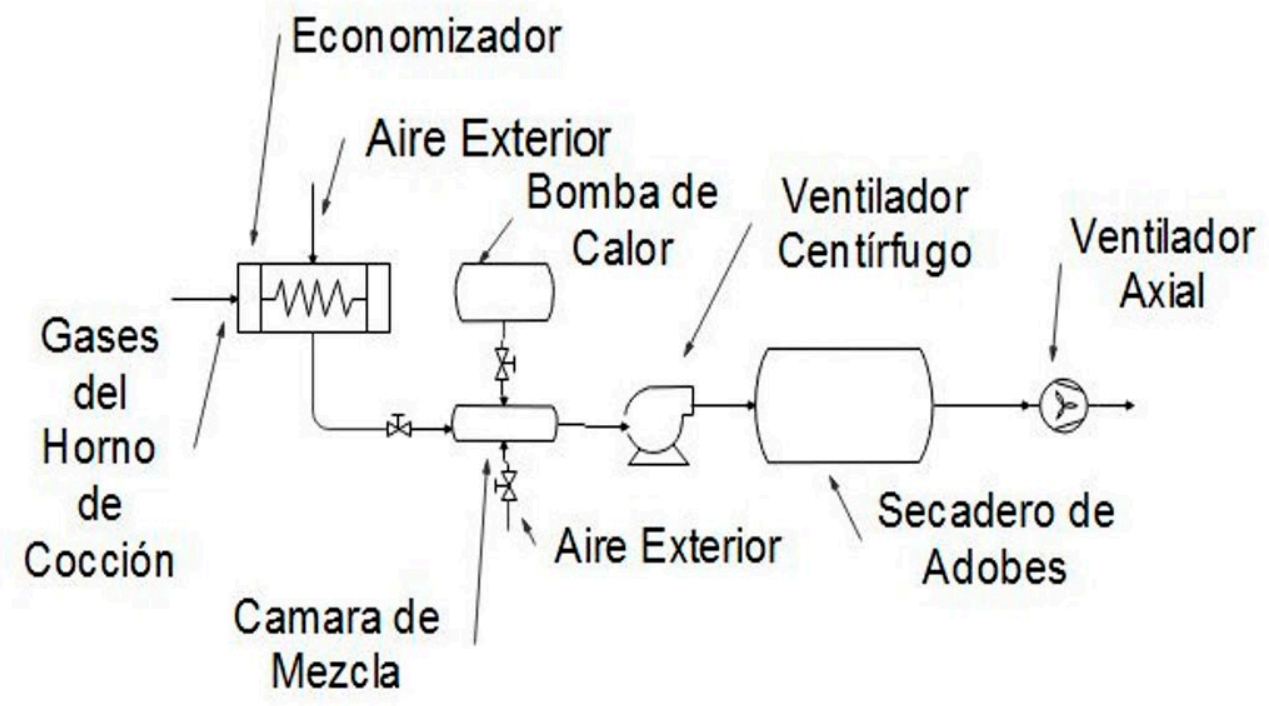

Figura 12: Esquema de calentamiento del aire 
En la figura 12 se observa el esquema de calentamiento del aire que se utiliza para el proceso de secado de los adobes. Cuando se realiza el proceso de cocción de los adobes, los gases de combustión procedentes de los hornos se los hace circular por el economizador, produciendo de esta manera el calentamiento del aire. Cuando el proceso de cocción de los adobes se encuentra interrumpido, el proceso de calentamiento del aire se realiza utilizando la bomba de calor. De esta forma se logra realizar el proceso de secado de los adobes sin interrupciones.

\section{Análisis energético utilizando los gases de combustión y/o la bomba de calor}

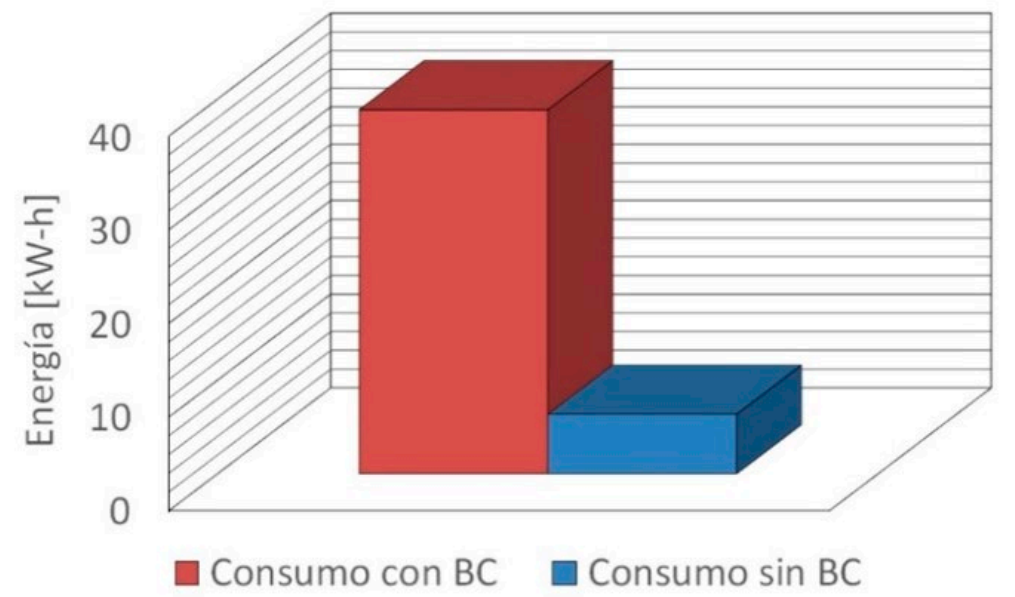

Figura 13: Análisis energético

La gráfica de la figura 13 muestra el análisis energético cuando se utiliza los gases de combustión para calentar el aire y la utilización de la bomba de calor cuando no se realiza el proceso de cocción de los adobes. Se observa que cuando se utiliza los gases residuales la energía consumida por el sistema de secado alcanza un valor de $6.34 \mathrm{~kW}$-h, mientras que cuando se emplea la bomba de calor la energía consumida es de $38.84 \mathrm{~kW}$-h. La utilización de los gases de combustión para calentar el aire, produce una reducción en el consumo del $84 \%$ de la energía total utilizada en el sistema de secado, en comparación cuando se utiliza la bomba de calor para realizar el mismo requerimiento.

\section{CONCLUSIONES}

Se realizó el estudio y desarrollo de un secadero de adobes de ladrillos macizos, utilizando los gases residuales del horno de cocción para calentar el aire y una bomba de calor cuando no se realiza el proceso de cocción de los adobes.

Se logró determinar que utilizando aire a una temperatura de $50^{\circ} \mathrm{C}$ en el secadero, el tiempo total del proceso de secado es de aproximadamente 60 horas. 
La cantidad de agua extraída de los 5000 adobes durante el proceso de secado es de aproximadamente $6265 \mathrm{~kg}$, lo que representa una pérdida de agua de 1250 gramos cada adobe.

Mediante el cálculo de la cantidad de agua extraída de los adobes, se logró determinar la potencia calorífica necesaria para calentar el aire a utilizar en el secadero.

Cuando el proceso de secado de los adobes se realiza con aire a una temperatura de $50^{\circ} \mathrm{C}$, al cabo de 60 horas, los adobes concluyen con un $12 \%$ de humedad referida a base seca.

Se realizó el proceso de simulación utilizando el programa SolidWorks 2016, para determinar el perfil de velocidad del aire caliente que circula por el secadero de adobes.

Cuando se utiliza los gases residuales, la energía consumida por el sistema de secado alcanza un valor de $6.34 \mathrm{~kW}-\mathrm{h}$, mientras que cuando se emplea la bomba de calor la energía consumida es de $38.84 \mathrm{~kW}$-h. Por lo tanto, el empleo de los gases de combustión para calentar el aire representa una reducción en el consumo del $84 \%$ de la energía total utilizada en el sistema de secado, en comparación cuando se utiliza la bomba de calor para realizar el mismo requerimiento.

Se realizó la selección del ventilador centrífugo y axial, economizador y bomba de calor, componentes importantes para el funcionamiento del secadero de adobes.

Se plantea como alternativa sustituir la bomba de calor mediante un hogar donde se queme leña o costaneros de aserraderos, de forma que los gases de combustión al pasar por el economizador caliente el aire a utilizar en el secadero.

\section{ABSTRACT}

The study and development of an adobe dryer of a solid brick factory in the area of Makalle (Chaco) is presented. Representative curves of the drying process were obtained for a volume of 5000 bricks inside the dryer. It was possible to determine for an approximate temperature of $50^{\circ} \mathrm{C}$ of the air entering the dryer, the amount of water extracted from the adobes is $6265 \mathrm{~kg}$ and the final time of the drying process is approximately 60 hours. In addition, the different components of the dryer were selected, such as: fans, economizer and heat pump.

\section{REFERENCIAS}

Moreno, F. El ladrillo en la construcción. España: Ediciones CEAC. 1981.

Spotorno R, Pochettino J, Figueredo G, García F. Ensayos Experimentales del Proceso de Secado de Adobe de Arcilla de la Zona de Makalle (Chaco). Actas del III CADI (Congreso Argentino de Ingeniería). Rcia, Chaco, Argentina. 7 al
9 de setiembre, 2016. http://www6.uniovi.es/usr/fblanco/Lección6. Secado pdf. Visitado 20/04/16. http://www.panelplac.com.ar.Visitado 19/09/17 http://www.gattisa.com.ar. Visitado 04/10/17 http://www.solerpalau.es. Visitado 10/10/17 https://www.climatecnica.com. Visitado 23/10/17 